\title{
ANTECEDENTES INTERNACIONALES Y NACIONALES DE LA PROMOCIÓN DE SALUD EN CHILE: LECCIONES APRENDIDAS Y PROYECCIONES FUTURAS
}

\author{
INTERNATIONAL AND NATIONAL BACKGROUND \\ OF THE CHILEAN HEALTH PROMOTION POLICY: LESSONS \\ LEARNED AND FUTURE PROYECTIONS
}

\author{
Mirta Crovetto M. (1), Fernando Vio del R. (2) \\ (1) Facultad de Ciencias Naturales y Exactas, Universidad de Playa Ancha, Valparaíso, Chile. \\ (2) Instituto de Nutrición y Tecnología de los Alimentos, Universidad de Chile. Santiago, Chile.
}

\begin{abstract}
The objective of this paper is to analyze different guidelines from rector institutions related with health risk factors, changes in lifestyles and its impact in chronic diseases in the last three decades. Technical documents and policy guidelines were reviewed since 1978 as well as World Health Organization (WHO) reports in health promotion and the prevention of chronic diseases. Chilean Ministry of Health, Universities and other institutions documents were reviewed at the national level and health promotion policies were analyzed. In conclusion, the Health Promotion Policy was incorporated in 1997 with the creation of the National Board for Health Promotion also named VIDA CHILE, in 1998, with important advances until 2003 in implementation of national and regional VIDA CHILE, including VIDA CHILE programs in 341 counties throughout the country. Plans of health promotion involving projects in food and nutrition, physical activity, tobacco and psychosocial and environmental problems were developed in each county. These accomplishments were possible thanks to an agreement between the Government of Chile and the academic sector. Since the year 2003, the Health Promotion Policy continued working at the regional and local level thanks to trained human resources, the support of the Ministry of Health and the local governments. At the world level, in the Bangkok meeting carried out in 2005, WHO incorporated the determinants of health with the purpose of decreasing inequities in the globalized world, modifying the concept of health promotion, and shifting the emphasis in the behavior of the population to a more structural vision of the determinants of health. The authors propose to integrate both concepts (health promotion and determinants of health) developing a State Policy similar to the policy utilized for the eradication of undernutrition in Chile, with a broad consensus and continuity on time, with an strategic agreement among Government, academic and private sector.
\end{abstract}

Key words: Health promotion, risk factors, chronic diseases, Vida Chile.

Este trabajo fue recibido el 9 de Octubre de 2008 y aceptado para ser publicado el 16 de Diciembre de 2008.

\section{INTRODUCCIÓN}

El incremento de las Enfermedades Crónicas No Trasmisibles (ECNT) en el mundo, asociadas a comportamientos y conductas relacionados con los factores de riesgo modificables, como la dieta, la actividad física, el consumo de tabaco, consumo excesivo de alcohol y factores psicosociales (stress, aislamiento y no participación en redes sociales, depresión y otros), caracterizan los estilos de vida occidental, tanto a nivel individual como poblacional. Ellos constituyen los principales desafíos en materia de salud pública, convirtiéndose hoy día en la principal carga de enfermedad a nivel mundial. Esto, a pesar de los esfuerzos realizados por organismos rectores de política en los países desde fines de los 70, que han señalado este problema en sus distintas publicaciones, comunicados e informes técnicos y especialmente, en reuniones internacionales de Promoción de la Salud. En estos informes y resoluciones se han orientado las 
acciones que se deben abordar en forma promocional y preventiva para enfrentar los factores de riesgo de las ECNT asociados a comportamientos. La demora en la toma de conciencia y el retardo en las acciones de política en los países, se ha acompañado con el aumento explosivo de estas enfermedades en el mundo, en especial de la obesidad, lo que nos lleva a reflexionar sobre las lecciones aprendidas en estos años.

Recientemente, en la $61^{\text {a }}$ Asamblea Mundial de la Salud celebrada en Ginebra, Suiza, del 19 al 24 de mayo del 2008 (1), se examinaron diversos aspectos de salud pública, en especial de las ECNT, como las cardiovasculares, cerebrovasculares y cánceres, a menudo relacionadas con el estilo de vida occidental, las que constituyen las principales causas de muerte en todo el mundo. En esta reunión fueron objeto de análisis, la prevención y control de las ECNT; la aplicación de la estrategia mundial en temas de salud pública, innovación y propiedad intelectual, en especial, el proyecto de estrategia mundial, plan de acción y estrategias para reducir el uso nocivo del alcohol y del cambio climático relacionado con salud (1).

La preocupación por las ECNT está presente en todos los informes que emanan de los organismos internacionales y sociedades científicas, ya que de mantenerse esta situación, el crecimiento de las ECNT será explosivo en los países de ingresos medios y bajos, impactando fuertemente la mortalidad en los próximos 25 años. Así, en el informe de salud 2008, presentado en la última Asamblea de Mundial de Salud se indica que para el año 2030 las muertes por cáncer, enfermedades cardiovasculares y accidentes de tráfico supondrán conjuntamente un $30 \%$ del total de las defunciones. "La diabetes y el asma están aumentando en todas partes. Incluso en los países con ingresos bajos se está viendo un aumento de la obesidad, especialmente en zonas urbanas, y en ocasiones, se ve desde la niñez", dijo Chan, Directora General de la Organización Mundial de la Salud (OMS).

Las ECNT son la principal causa de muerte y discapacidad prematuras en la gran mayoría de los países de América Latina y del Caribe, señala otro documento de la OPS (2) que indica que la carga de morbilidad por ECNT en las Américas puede ser mayor de lo que indican las estadísticas, considerando que el número de notificaciones de fallecimientos en la Región puede ser inferior al real.

Las ECNT más frecuentes y con una mayor importancia en la Región de las Américas son las cardiovasculares (incluida la hipertensión), el cáncer, las enfermedades respiratorias crónicas y la diabetes. Como ejemplo, se calcula que en el primer decenio del siglo XXI fallecerán aproximadamente 20 millones de perso- nas víctimas de enfermedades cardiovasculares.

El objetivo de este artículo es analizar las orientaciones técnicas y directrices que han entregado los organismos rectores sobre los factores de riesgo en salud, los cambios en los estilos de vida y su impacto en las ECNT en las últimas tres décadas. Además, se analizan las políticas aplicadas en Chile de acuerdo a estos lineamientos internacionales, y se hacen propuestas para enfrentar el tema.

\section{MATERIAL Y MÉTODO}

Revisión bibliográfica de documentos técnicos y directrices de política en salud pública internacionales desde 1978 a la fecha, en áreas de promoción de la salud y de prevención de ECNT, de tendencias en países desarrollados y en desarrollo elaborados por grupos técnicos de alto nivel y especialización de distintos países del mundo, convocados por la Organización de Naciones Unidas (ONU), Organización Mundial de la Salud (OMS) y Organización Panamericana de la Salud (OPS), Organización para la Agricultura y la Alimentación de las Naciones Unidas (FAO). A nivel nacional, se revisaron documentos técnicos del Ministerio de Salud (MINSAL), Universidades y entidades relacionadas. El análisis de documentos técnicos, conferencias y estrategias a nivel internacional y en Chile, servirán para reflexionar sobre la salud, la promoción de la salud y la relación con el incremento de las ECNT en el mundo. Esta revisión se hará en base a los hitos en Promoción de la Salud, documentos técnicos relacionados con alimentación y nutrición y los informes de salud en el mundo, para terminar con lo ocurrido en Chile en este ámbito.

\section{RESULTADOS}

\section{Hitos en promoción de la salud}

Desde 1978, con la Declaración de Alma Ata, en la que se estableció el lema "Salud para todos en el año 2000" (3), la promoción de la salud ha sido un eje de orientación para la creación de condiciones que mejoren y promuevan la salud como un derecho básico universal, situándola como una de las mejores inversiones para lograr el desarrollo económico y social de los países. En ese sentido, se hizo énfasis en la Estrategia de Atención Primaria de Salud (APS) para solucionar los problemas de salud asociados a carencias.

Posteriormente, en 1986, se celebra en Ottawa, Canadá, la Primera Conferencia Internacional de Promoción de la Salud llamada "Iniciativa en pro de una nueva acción de salud pública", con la publicación de la Carta de Ottawa, orientada también al objetivo "Salud para todos en el año 2000". En ella se analizan los progresos 
alcanzados desde la declaración de Alma Ata en APS y se pone énfasis en una nueva concepción de salud pública, desde el concepto de promoción basado en el autocuidado y la acción intersectorial en salud.

Se define el concepto de promoción de la salud indicando que la salud es una responsabilidad no sólo del sector salud y se plantea que es parte del bienestar de la población, involucrando a todos los sectores. Para su logro, se requiere de la elaboración de una política pública sana, la creación de ambientes saludables, el reforzamiento de la acción comunitaria, el desarrollo de las aptitudes personales, la reorientación de los servicios de salud y el compromiso a favor de la promoción de salud de quiénes la promueven. Hace un llamado a la acción internacional, identificando acciones claves y estrategias básicas para lograr la "Salud para todos" establecida por la OMS en Alma Ata. Al finalizar la reunión, se genera el documento conocido como la Carta de Ottawa (4).

La Segunda Conferencia Internacional de Promoción de la Salud se celebró en Adelaida, Australia en 1988, con el slogan "Políticas públicas favorables a la salud". En esta reunión se declaró la importancia de la construcción de políticas públicas saludables, señalando que éstas deben ser abordadas por todos los sectores para actuar sobre los determinantes de la salud y reducir las inequidades sociales y el acceso equitativo a bienes, servicios y a la atención en salud. Se identificaron cuatro áreas claves para una acción inmediata en salud pública: i) apoyar la salud de la mujer; ii) mejoramiento de la seguridad alimentaria de la población; iii) reducción del consumo del tabaco y del alcohol, y iv) la creación de ambientes saludables para la salud. Se termina con las "Recomendaciones de Adelaida sobre políticas públicas favorables a la Salud" (5).

En 1991, se celebra en Sundsvall, Suecia, la Tercera Conferencia Internacional de Promoción de la Salud titulada: "Podemos hacerlo", la que se centró específicamente en promover el concepto de vida saludable y la creación de ambientes favorables para la salud, reflejadas en la Declaración de Sundswall (6).

En 1992, se realiza la Conferencia Internacional de Promoción de la Salud, en Santa Fe de Bogotá y se adopta la Declaración de Promoción de la Salud en América Latina. Se analizó la creación de condiciones que garanticen el bienestar general como propósito fundamental del desarrollo. Instó a renovar el compromiso con la solidaridad y la equidad en lo atingente a salud. Deploró la violencia y apeló a la voluntad política para modificar las condiciones sociales con el objeto de que la marginalidad, la desigualdad, el abuso y el daño ambiental sean inadmisibles (7).

En Francia, en 1994, se realiza el Seminario In- ternacional sobre Políticas, Estrategias y Estructuras Nacionales de Promoción de la Salud. Los acuerdos más importantes fueron la movilización de recursos y apoyo a la Promoción de la Salud y la creación de condiciones para políticas, estructuras y roles que la favorezcan, incorporando el tema político - estructural (8).

En 1997 se realiza en Yakarta, Indonesia, la Cuarta Conferencia Internacional sobre Promoción de la Salud: "Nueva era, nuevos actores; adaptar la promoción de la salud al siglo XXI". Es la primera conferencia organizada en un país en desarrollo en que participa el sector privado en la promoción de la salud. Se reflexiona sobre lo aprendido con respecto a la promoción de la salud, propone reconsiderar los factores determinantes de la salud y señalar los obstáculos y las estrategias necesarias para resolver las dificultades de promoción de la salud en el siglo XXI. Reitera el compromiso internacional con las estrategias de la Carta de Ottawa y hace énfasis en la participación social en el ámbito comunitario y las capacidades de las personas para la promoción de la salud. De esta reunión surge la Declaración de Yakarta (9).

En 1998 aparece la Resolución sobre Promoción de la Salud adoptada durante la LI Asamblea Mundial de la Salud que insta a todos sus miembros a: i) promover la responsabilidad social para la salud; ii) incrementar la inversión para el desarrollo de la salud; iii) consolidar y expandir la "corresponsabilidad en salud"; iv) incrementar la capacidad comunitaria y empoderar al individuo en materia de salud; v) fortalecer las consideraciones de los requerimientos en salud y promoción en todas las políticas, y vi) adoptar un enfoque basado en evidencia para las políticas de promoción de la salud y sus prácticas, utilizando metodologías cuantitativas y cualitativas. Hace un llamado a las instituciones públicas y organizaciones no gubernamentales e intergubernamentales, privadas, donantes y comunidad internacional a que apoyen la implementación de las estrategias y establezcan una red global en apoyo a la promoción de salud, e insta al desarrollo de espacios saludables y a la implementación de estrategias de promoción durante todo el ciclo vital, priorizando a los grupos vulnerables (10).

En México, en el año 2000, se realiza la Quinta Conferencia Internacional de Promoción de Salud: "Promoción de la Salud hacia una mayor equidad". El objetivo fue enfatizar el aporte de la promoción de la salud en las políticas, los programas y proyectos de salud y calidad de vida, promoviendo los Planes Nacionales de Promoción de la Salud. Se instó a apoyar la investigación para conocer prioridades y la movilización de recursos financieros y operacionales para elaborar, aplicar, vigilar y evaluar los planes de acción nacionales y establecer o fortalecer redes nacionales e internacionales que 
promuevan la salud.(11). Se avanzó en el desarrollo de las cinco prioridades para el Siglo XXI en Promoción de la Salud, que fueron identificadas en Yakarta y confirmadas en la Resolución sobre la Promoción de la Salud adoptada por la Asamblea Mundial de la Salud en mayo de 1998.

En Bangkok, Tailandia 2005, se realizó la Sexta Conferencia Mundial de Promoción de la Salud, titulada «Los determinantes de la salud en relación con las políticas y las alianzas para la acción sanitaria» orientada a reducir las desigualdades en materia de salud en un mundo globalizado mediante el fomento de la salud (12). Aquí se introduce con mucha fuerza el tema de los determinantes de la salud, como son los conceptos de equidad y condiciones socio-económicos, género, situación laboral, vivienda, relacionados con la salud.

El resumen de las reuniones se señala en el cuadro 1.

\section{Informes de la salud en el mundo de la OMS}

Desde 1995, los informes de la OMS son la principal publicación sobre salud en el mundo. Estos presentan una evaluación de la salud mundial a cargo de expertos, incluidas estadísticas sobre todos los países, con el análisis de un tema particular. El objetivo principal de éstos, es proporcionar a los países, los organismos donantes, las organizaciones internacionales y otras entidades, la información necesaria para ayudar a tomar decisiones de política y de financiamiento. Algunos de estos informes se orientan principalmente hacia las ECNT y los factores re riesgo.

Los principales informes sobre la salud en el mundo son:

- 1995 - Reducir las desigualdades. Se pone el énfasis en la pobreza y las desigualdades entre ricos y pobres y su impacto en la salud (13).

- 1996 - Combatir las enfermedades y promover el desarrollo (14).

- 1997 - Vencer el sufrimiento, enriquecer a la humanidad. Hace un llamado de atención a la propagación de las ECNT, en especial, en los sectores más desposeídos, las que combinadas con las enfermedades infecciosas, imponen a los países en desarrollo una doble carga de morbilidad. Aboga por una actuación integral y global que tenga en cuenta todos los determinantes de la mala salud (15).

- 1998 - La vida en el siglo XXI. Coincidiendo con el cincuentenario de la OMS, se hace un repaso de las tendencias sanitarias registradas durante los

\section{CUADRO 1}

\section{Resumen reuniones internacionales en Promoción de Salud. 1978-2005}

1978 Alma Ata

1986 Ottawa, Canadá

1988 Adelaida, Australia

1991 Sundsvall, Suecia

1992 Santa Fé, Bogotá

Conferencia Internacional de

Promoción de la Salud

1994 Paris, Francia: Seminario Internacional sobre las Políticas, Estrategias y Estructuras Nacionales de Promoción de la Salud

1997 Yakarta, República de Indonesia

1998 LI Asamblea Mundial de la Salud. Resolución sobre la Promoción de la Salud

2000 Santa Fe, México

2005
Atención Primaria de Salud (3)

Promoción de Salud (Conceptos) (4)

Políticas públicas favorables a la Promoción de Salud (5)

Crear ambientes favorables para la Promoción de Salud (6)

Declaración de Promoción de la Salud

en América Latina (7)

Incorporación de lo político-estructural en la

Promoción de Salud (8)

"Nueva era, nuevos aires"; incorpora al sector privado a la Promoción de Salud (9)

Hace un llamado a la comunidad internacional a implementar y apoyar las acciones en Promoción de Salud (10)

Se establecen Planes Nacionales de Promoción de Salud para los países e América Latina (11)

Determinantes de la salud en las alianzas para la acción sanitaria: disminución desigualdades en el mundo globalizado (12) 
cinco decenios anteriores y las enseñanzas obtenidas durante ese periodo, incluyendo la evolución de la salud hasta el año 2025, de la esperanza de vida, las condiciones sanitarias y los instrumentos para mejorarlas (16).

- 1999 - Cambiar la situación. Se describen los logros alcanzados durante el siglo XX y los desafíos asociados. Se proponen una serie de estrategias para propiciar un cambio hacia una mejor salud en el siglo XXI. El informe afirma que la salud debe ocupar un lugar central dentro del programa mundial de desarrollo y subraya la adhesión de la OMS a ese objetivo (17).

- 2000 - Mejorar el desempeño de los sistemas de salud. Analiza y compara los sistemas de salud del mundo entero, los factores que explican el funcionamiento de los sistemas de salud y las orientaciones para evaluar el desempeño y lograr mejoras con los recursos disponibles (18).

- 2002 - Reducir los riesgos y promover una vida sana. Describe la carga mundial de morbilidad, discapacidad y mortalidad actual que se puede atribuir a un número seleccionado de riesgos más importantes para la salud humana. Indica qué proporción de esa carga se podría reducir en los próximos 20 años mediante la atenuación de esos factores de riesgo y la promoción de la salud (19). Es primera vez que aparece claramente como política OMS, el tema de reducción de factores de riesgo de ECNT y la promoción de la salud.

- 2003 - Forjemos el futuro. En el Capítulo 6: "Epidemias mundiales desatendidas: tres amenazas crecientes", señala que la carga de mortalidad y discapacidad atribuible a las ECNT, en particular las afecciones cardiovasculares, supera en los países en desarrollo la carga impuesta por enfermedades transmisibles presentes desde hace tiempo. Para hacer frente a este fenómeno, que se denomina «doble carga de enfermedad», se propone una «doble respuesta», consistente en combinar, dentro de sistemas integrales de atención de salud, la prevención y el control tanto de las enfermedades transmisibles, como de las ECNT (20).

- 2006 - Colaboremos por la salud. Presenta una evaluación realizada por expertos acerca de la crisis de personal sanitario que atraviesa el mundo, así como varias propuestas para abordarlo (21).

- El Informe sobre la salud en el mundo 2007 - Un porvenir más seguro: Protección de la salud pública mundial en el siglo XXI-. Marca un hito en la historia de la salud pública y señala lo que podría ser uno de los mayores adelantos realizados en medio siglo para alcanzar la seguridad sanitaria (22).

- El Informe sobre la Salud en el mundo 2008, se dedica a una de las esferas prioritarias de la OMSLa revigorización de la Atención Primaria de Salud (APS), volviendo a lo que la OMS propuso 30 años atrás en la Conferencia Internacional sobre atención primaria de ALMAATA. Con este informe pareciera cerrarse el círculo Atención Primaria de Salud-Promoción de la Salud y- Atención Primaria en Salud. (23)

\section{Documentos técnicos}

Se examinan algunos informes relacionados con dieta y actividad física elaborados en los últimos años y documentos técnicos orientados hacia factores de riesgo de las ECNT y el rol de la promoción de conductas saludables en alimentación, actividad física, consumo de tabaco, alcohol y factores psicosociales. En 1990, el informe $\mathrm{N}^{\mathrm{o}} 797$ "Dieta, nutrición y prevención de enfermedades crónicas" indicaba que éstas eran en gran medida enfermedades prevenibles, analizando la estrecha vinculación existente entre los factores alimentarios y varias afecciones crónicas, como son la cardiopatía coronaria, la hipertensión, los accidentes cerebrovasculares, el cáncer, la diabetes y la osteoporosis (24).

Posteriormente, la Conferencia Internacional sobre Nutrición (CIN), organizada por la FAO/OMS en 1992, identificó específicamente la necesidad de prevenir y controlar los crecientes problemas de salud pública que representan las ECNT, promoviendo dietas apropiadas y modos de vida sanos (25).

En mayo del 2002, por una resolución adoptada por sus Estados Miembros en la Asamblea Mundial de la Salud, la OMS y la FAO convocan a un grupo de expertos a la Consulta de Expertos de la OMS/FAO sobre Régimen Alimentario, la Nutrición y la Prevención de las Enfermedades Crónicas (26), que emite su informe el 2003, el cual señala que una dieta saludable basada en el bajo consumo de alimentos muy energéticos, ricos en grasas saturadas, azúcar y sal y en la ingesta abundante de frutas y hortalizas, así como un modo de vida activo, figuran entre las principales medidas para combatir las enfermedades crónicas. Se hacen recomendaciones sobre régimen alimentario y actividad física destinadas a los gobiernos, con miras a enfrentar el creciente número de muertes por enfermedades crónicas, que representan la mayor carga de enfermedad en el mundo.

Con este informe, la OMS establece la "Estrategia Mundial sobre Régimen Alimentario, Actividad Física y Salud 2005" que tiende a implementar políticas y programas de alimentación y actividad física en los países de América Latina (27). 
Posteriormente, se realizan diversas reuniones internacionales en dieta y actividad física, una de ellas, organizada por OPS/OMS en Washington en abril 2007, discutió el problema de los ácidos grasos trans en Las Américas. Como consecuencia de esta reunión, se resolvió crear el Grupo de Trabajo OPS/OMS “Las Américas Libres de Grasas Trans" y se redactó un documento de Conclusiones y Recomendaciones del Grupo, donde se dan los lineamientos generales de las acciones para lograr el objetivo que refleja su nombre (28).

Otra iniciativa, la constituye el programa de promoción de consumo de frutas y verduras " 5 al día", que se inicia en California en 1991, apoyado por el Instituto Nacional del Cáncer de los Estados Unidos, que luego se extiende por todo el mundo apoyado por la OMS. En el año 2003 se realiza otra reunión en Ginebra cuyas propuestas se oficializan en un documento realizado en Christchurch, Nueva Zelanda, el año 2004 (29). Por otra parte, el Instituto Nacional del Cáncer de los Estados Unidos y la Asociación de Cardiología elaboraron en 1997 un documento sobre Dieta y Cáncer (30) que se revisó 10 años después con la evidencia proveniente de más de 7000 trabajos científicos y la información de los sujetos sobrevivientes de cáncer, el cual fue dado a conocer a la comunidad científica a nivel mundial en noviembre 2007 (31), entregando recomendaciones de cómo prevenir el cáncer, donde la dieta rica en vegetales y la actividad física son los pilares fundamentales.

El resumen de estos documentos se describe en el cuadro 2 .

\section{Documentos nacionales}

Chile, experimenta una rápida transición epidemiológica y nutricional entre la década del 70 y los 90 (32 - 34). De acuerdo a estos cambios, se comienza a trabajar en nuevas orientaciones de política y salud, realizándose en 1995 el Estudio de Carga de Enfermedad (35) que demostró que el 75\% de los Años de Vida Saludable (AVISA) perdidos en Chile se debían a ECNT y a problemas de salud mental. Con estos antecedentes, se cambiaron las prioridades existentes en salud, que estaban principalmente enfocadas al área materno-infantil y a las enfermedades infecciosas.

En 1998, se crea el Consejo Nacional para la Promoción de la Salud Vida Chile, como una de las respuestas a las necesidades de la población y a las demandas del perfil epidemiológico del país, elaborándose el Plan Nacional de Promoción de la Salud con prioridades precisas, siguiendo los lineamientos de la OMS $(36,37)$. Estas prioridades establecen que los cuatro grupos de enfermedades que deben ser abordados desde la Promoción de Salud son las enfermedades cardiovasculares, problemas de salud mental, accidentes y cáncer, las que deben enfrentarse desde sus cinco condicionantes principales: alimentación, actividad física, tabaco, factores protectores psicosociales y factores ambientales, que se implementan en tres espacios o lugares donde se desarrolla la promoción: establecimientos educacionales (jardines infantiles, escuelas y Universidades), lugares de trabajo y comunas (cuadro 3). El Plan Nacional de Promoción de la salud, se implementa mediante un modelo de gestión descentralizado e intersectorial, basado en la participación social y en el fortalecimiento de los Gobiernos Regionales y Locales.

El Ministerio de Salud, en conjunto con VIDA CHILE, además de las prioridades, se compromete con metas de impacto sobre los condicionantes (alimentación, actividad física, tabaco) y los factores protectores psicosociales y ambientales de la salud en noviembre del año 2000, las que se constituyeron en las metas sanitarias para la década (38).

Para llevar adelante este plan se realizan intervencio-

\section{CUADRO 2}

\section{Documentos técnicos sobre Alimentación, Nutrición y Actividad Física 1990-2007}

1990 Dieta, Nutrición y Prevención de Enfermedades Crónicas. Informe Técnico No 797 (24)

1992 Conferencia Internacional sobre Nutrición (25)

2003 Dieta, Nutrición y Prevención de Enfermedades Crónicas. Documento TRS 916 (26)

2005 Estrategia Mundial sobre Régimen de Alimentación, Actividad Física y Salud (27)

2005 Marco para la Promoción de Frutas y Verduras (29)

2007 Las Américas Libres de Grasas Trans (28)

2007 Dieta y Cáncer (30)) 
nes para la formación de recursos humanos en Promoción de la Salud a distintos niveles organizacionales, desde mediados de los años 90 hasta el año 2003 en forma continua, generando un potencial en recursos humanos que ha dado sustentabilidad al enfoque de salud basado en acciones promocionales. Parte de estas experiencias de capacitación se registran en dos publicaciones $(39,40)$ y se elabora el manual Salud sin Tabaco (41). En 1999 se realiza el Primer Congreso Nacional de Promoción de la Salud con más de dos mil participantes y se firma el compromiso de Huechuraba (42). El libro resumen de este congreso da cuenta del compromiso de los equipos de salud y de las diversas acciones implementadas a nivel nacional en un corto período, que indican un cambio en el abordaje de la salud, empezando a visualizar las acciones promocionales como un eje importante para detener los factores de riesgo (43).

En el año, 2002, se realiza el II Congreso Chileno de Promoción de la Salud (44). En el año 2000, se aplica la primera Encuesta Nacional de Calidad de Vida para tener una línea base de las condicionantes en salud y evaluar el impacto de las acciones en promoción para comparar resultados y avances, la que se vuelve a aplicar en el año $2006(45,46)$. En el año 2003, se aplica la Primera Encuesta Nacional de Salud (47).

Paralelamente, se desarrollan una serie de documentos técnicos para apoyar los lineamientos de política, entre ellos, las guías dirigidas a la población en condicionantes de salud. Así, en 1997, surgen los mensajes de las Guías Alimentarias para la población chilena (48); el 2003 se publican las Guías para una Vida Activa (49) y se presenta un modelo de intervención promocional en atención primaria haciendo énfasis en la Consejería de Vida Sana (50). En el 2005 -2006 se presentan las Guías para una Vida Sana que dan orientaciones integradas sobre estilos de vida para apoyar cambios conductuales en alimentación, actividad física, tabaco y factores protectores psicosociales. En esta oportunidad se revisan y actualizan los mensajes de las Guías Alimentarias de 1997 (51).

En el año 2006 se lanza la Estrategia Global contra la Obesidad (EGO Chile) que reúne al ámbito público y privado y sociedades científicas y colegios profesionales, en una declaración de intenciones para enfrentar la obesidad (52). En este sentido, se desarrollan orientaciones para implementar estrategias de ambientes favorables al interior de los lugares de trabajo y de educación, elaborándose las Guías de Vida Sana en la Empresa en el 2004 (53) y la Guía de Universidades Saludables en el 2006 (54).

\section{Metas en promoción de salud}

Estas metas consideraban una disminución de la prevalencia de obesidad de un $10 \%$ a un $7 \%$ en los niños de 2 a 5 años que asisten a los jardines infantiles de la Junta Nacional de Jardines Infantiles (JUNJ); de $16 \%$ a $12 \%$ en los escolares de primer año básico que ingresan al sistema escolar, registrados por la Junta Nacional de Auxilio Escolar y Becas (JUNAEB) y de un $32 \%$ a un $28 \%$ en las embarazadas controladas en los servicios de salud. En actividad física, se estableció reducir el sedentarismo de un $91 \%$ de la población a un $84 \%$. En tabaquismo, se planteó reducir la prevalencia en escolares de octavo año básico de un $27 \%$ a un $20 \%$; en mujeres en edad fértil de un $45 \%$ a un $40 \%$ y de un $40 \%$ a $30 \%$ en la población general. (cuadro 4 ).

Al término del 2007, no se han producido los resultados esperados: sólo se ha logrado una mantención en la obesidad en niños que se atienden en el sector público en salud (que no estaban en las metas iniciales) y afecta a un 7,2\%; y en los niños de 2 a 5 años atendidos en la JUNJI se mantiene en un 10\%. (9,6\% el 2007). En los

\section{CUADRO 3}

Plan Nacional de Promoción de Salud. Prioridades sanitarias; condicionantes y estrategias.1998

\begin{tabular}{llll}
\hline $\begin{array}{l}\text { Prioridades } \\
\text { sanitarias }\end{array}$ & $\begin{array}{l}\text { Condicionantes } \\
\text { de Salud }\end{array}$ & $\begin{array}{l}\text { Espacios de } \\
\text { promoción }\end{array}$ & $\begin{array}{l}\text { Estrategias } \\
\text { generales }\end{array}$ \\
\hline $\begin{array}{l}\text { Cardiovasculares } \\
\text { Salud Mental }\end{array}$ & $\begin{array}{l}\text { Alimentación } \\
\text { Actividad Física }\end{array}$ & $\begin{array}{l}\text { Establecimientos educacionales } \\
\text { Lugares de Trabajo }\end{array}$ & $\begin{array}{l}\text { Regulación y Legislación } \\
\text { Información, comunicación social }\end{array}$ \\
Cáncer & Tabaco & Comunas & Formación de Recursos Humanos \\
& Factores Psicosociales & & Participación Social \\
\hline Fuente: Ministerio de Salud. Plan Nacional de Promoción de la Salud. Santiago, Chile 1999 & Reorientación de Servicios de Salud \\
\hline
\end{tabular}


escolares que ingresan al primer año básico la obesidad ha aumentado llegando al 19,4\% en el 2006 una de las más altas del mundo; la situación de la embarazadas se mantiene en $32,6 \%$, según los últimos datos medidos por la referencia MINSAL el 2004, antes de que fuese cambiada a otra referencia con IMC para la edad gestacional, que se puso en práctica el año 2005 (55).

En actividad física, la Encuesta Nacional de Salud 2003, indicó que el sedentarismo se mantenía alrededor del $90 \%$ de la población, cuya condición prácticamente se mantiene en la Encuesta del 2006 con un $88 \%$ de sedentarismo. Estas cifras son coincidentes con los resultados de la Encuesta Nacional de Hábitos de Actividad Física y Deportes en la población chilena de 18 y más años, realizada por Chile Deportes durante el año 2006, que confirma datos anteriores y revela que el $87 \%$ de la población es sedentaria, es decir 9 de 10 chilenos no realizan actividad física tres veces por semana, mínimo 30 minutos cada vez (56).

En tabaquismo, según la ENS 2003 la prevalencia de fumadores mayores de 17 años es de un $42 \%$ y en mujeres en edad fértil de un $47 \%$ en el grupo de 17 a 24 años y de un $44 \%$ en el grupo de 25 a 44 años. Sin embargo, en el último estudio del CONACE 2007, se aprecia un descenso en el consumo de tabaco en la población escolar de $42 \%$ a 35\% en el bienio 2005-2007, y de un $27 \%$ a $20 \%$ en los de octavo año básico (57). Esta es el primer descenso significativo que se aprecia en el país con la puesta en práctica de la Ley del Tabaco.

La falta de impacto de las acciones realizadas en Promoción de Salud para disminuir las metas puede deberse a tres factores: a) El impacto del cambio de la transición epidemiológica que instaló a las ECNT en el principal problema de salud pública, sin las adecuaciones y capacidades técnicas oportunas para enfrentar la nueva realidad epidemiológica, con políticas que a pesar de ello, hacen énfasis y privilegian a la enfermedad por sobre la mantención de la salud; al desconocimiento inicial del impacto de los cambios conductuales en los condicionantes de la salud, variable que escapa al control directo del personal de salud, lo que dificulta aun más el abordaje de éstos e indica, por tanto, que las acciones de promoción de la salud deben ser mantenidas y sustentables en el largo plazo, con mayor número de recursos humanos y financieros, con respaldo legislativo y reglamentario, situación que no ha sucedido; b) la interrupción de la política de Promoción de Salud desde el 2003 en adelante por la prioridad dada por MINSAL al programa

\section{CUADRO 4}

Metas Sanitarias al 2010. Evolución. 2000-2007

\begin{tabular}{|c|c|c|c|c|c|c|}
\hline Condicionantes & Metas & 2000 & 2010 & 2005 & 2006 & 2007 \\
\hline \multirow[t]{4}{*}{ Obesidad } & $\begin{array}{l}\text { Preescolares controlados en } \\
\text { el Sistema Público de Salud }\end{array}$ & & & & 7,2 & $7,2 \%$ \\
\hline & Preescolares JUNJI* & $10 \%$ & $7 \%$ & $10,6 \%$ & $10,3 \%$ & 9,8 \\
\hline & $\begin{array}{l}\text { Escolares Primer Año } \\
\text { Básico JUNAEB }\end{array}$ & $16 \%$ & $12 \%$ & $18,5 \%$ & 19,4 & $21,0 \%$ \\
\hline & Embarazadas Servicios de Salud & $32 \%$ & $28 \%$ & $32,6 \%$ & & \\
\hline Actividad Física & $\begin{array}{l}\text { Sedentarismo población } \\
\text { mayor } 15 \text { años }\end{array}$ & $91 \%$ & $84 \%$ & $89 \% * *$ & $88 \% * * *$ & \\
\hline \multirow[t]{3}{*}{ Tabaquismo } & Escolares octavo año básico & $27 \%$ & $20 \%$ & $26,5 \% * * * *$ & & $20,4 \%$ \\
\hline & Mujeres edad fértil (17-44 años) & $45 \%$ & $40 \%$ & $\begin{array}{l}47 \%(17-24)^{*} \\
44 \%(25-44)^{*}\end{array}$ & & \\
\hline & Población General & $40 \%$ & $30 \%$ & $42 \% *$ & & \\
\hline \multicolumn{7}{|c|}{$\begin{array}{l}\text { *GESPARVU: JUNJI, } 2008 \\
\text { **Encuesta Nacional de Salud 2003. Resultados entregados el } 2004 \\
\text { *** Encuesta Nacional de Hábitos de Actividad Física y Deportes Encuesta Nacional de Salud } 2006 . \\
\text { **** Encuesta CONACE 2005. Resultados entregados en octubre } 2006 \\
\text { ***** Encuesta CONACE 2006 } \\
\text { ****** Encuesta CONACE } 2007\end{array}$} \\
\hline
\end{tabular}


AUGE y la aparición del tema de los determinantes en salud, que disminuyó las acciones en Promoción de Salud y desvinculó las acciones que se estaban realizando en forma integrada, y c) la falta de coordinación de las políticas actuales en Promoción de Salud. Por una parte, existe VIDA CHILE funcionando en Regiones; a ello se agregó la estrategia EGO de MINSAL; se creó la Corporación 5 al día Chile, con apoyo del Ministerio de Agricultura, MINSAL, FAO, OMS/OPS, PNUD y sector privado para promover el consumo de frutas y verduras en el país, y el programa NutriRSE de Acción Responsabilidad Social Empresarial, con empresas privadas apoyando escuelas básicas en temas de alimentación y actividad física para prevenir la obesidad, siguiendo el modelo de Casablanca (58)

\section{DISCUSIÓN Y CONCLUSIONES}

De acuerdo a lo descrito en este artículo, se observa que el tema de Promoción de Salud es relativamente nuevo en la temática de la Salud Pública internacional. Los primeros documentos son de la década del 80 y solamente a mediado de los 90 se aprecian cambios en las políticas nacionales de algunos países $(7,8)$. En este período, aparece con mucha fuerza en la OMS el concepto de transición epidemiológica y nutricional con la doble carga de enfermedad a partir de los estudios de carga y enfermedad desarrollados por Murray y López (59).

El primer estudio nacional se desarrolló en México en 1990 y en Chile en 1995 con los datos de mortalidad de 1993. En el estudio de Chile, se demostró claramente que el $75 \%$ de la carga de enfermedad la constituían las ECNT. Posteriormente, Murray y López demostraron que las prioridades de la OMS no correspondían a la carga de enfermedad del mundo, en que la principal carga la constituían las ECNT y no las infecciosas (60).

Estos antecedentes hicieron que en Chile se cambiaran las prioridades en Salud y la estrategia de Promoción de Salud fue incorporada rápidamente en las políticas nacionales desde 1997 en adelante, creándose el Consejo Nacional de Promoción de Salud o VIDA CHILE en 1998 (36). Este paralelismo entre una política internacional iniciada en 1986 con la declaración de OTTAWA e implementada en Chile en 1998, muestra lo importante que fue la adecuación de políticas internacionales al contexto nacional.

En Chile, los avances en Promoción de Salud que se realizaron entre 1998 y el año 2003 fueron muy importantes. En este período, se logró consolidar una estructura nacional con un VIDA CHILE nacional que se reunía mensualmente y la instalación de los VIDA CHILE regionales en todo el país (con excepción de la Región Metropolitana) y con los VIDA CHILE locales en 341 comunas del país. En cada una de estas comunas funcionaron planes comunales de Promoción de la Salud con proyectos en alimentación, actividad física, tabaco, temas psicosociales y ambientales. Esto se logró gracias a una alianza estratégica entre el gobierno, principalmente a través del MINSAL, Chile Deportes, Educación, y el sector académico. Para ello, se contó con el apoyo del gobierno de Canadá que creó tres Centros Académicos de Recursos en Promoción de la Salud en las Universidades Austral, de Concepción y el INTA de la Universidad de Chile. A través de estos centros se logró capacitar a las autoridades regionales de salud en 1998, al 70\% de los municipios del país entre 1999 y 2001 y a todos los VIDA CHILE regionales el 2001-2002, lo que dejó instalada una masa crítica de recursos humanos capacitados en los temas de Promoción de Salud.

\section{CUADRO 5}

\section{Cronología de la Promoción de Salud en Chile. 1995-2006}

1996 Informe Estudio de Carga de Enfermedad (35)

1997 Redefinición de Prioridades en Salud (36)

1998 Estrategia de Promoción de Salud (37)

1999 I Congreso de Promoción de Salud (43)

$2000 \quad$ Metas 2000-2010 (38)

2000 I Encuesta de Calidad de Vida y Salud (45)

2002 II Congreso de Promoción en Salud (44)

2003 I Encuesta Nacional de Salud (47)

2006 II Encuesta de Calidad de Vida y Salud (46) 
Desde el año 2002 en adelante, la política de salud se focalizó principalmente en el Plan de Acceso Universal con Garantías Explícitas en Salud (Plan AUGE) y la Promoción de Salud dejó de tener la prioridad que se le dio anteriormente a nivel nacional. Sin embargo, continuó funcionando en el nivel local y regional, en gran medida debido a los recursos humanos capacitados y al apoyo del MINSAL y los gobiernos locales. Además, se mantuvo el apoyo técnico desde las Universidades, lo que se manifiesta en el desarrollo de guías para la población (48 - 51) y materiales educativos.

A nivel mundial, la OMS continuó planteando la importancia de la Promoción de Salud, pero a partir del año 2005 en la reunión de Bangkok (12) incorpora con mucha fuera los determinantes de la salud con el objeto de disminuir las desigualdades en el mundo globalizado. Esto modifica en parte el concepto de promoción de salud, que enfatiza principalmente cambios de conducta en la población, a una visión más estructural de los determinantes de salud que incorpora todos los factores que pueden tener una relación con la salud: inequidad, pobreza, condiciones laborales, temas de género, vivienda, medio ambiente. Es decir, la mirada sociológica- estructural de los determinantes sociales y condiciones materiales pasa a tener preponderancia sobre los temas relacionados con los hábitos y conductas de la población (61). En este sentido, lo que se debe hacer es integrar ambas miradas, ya que ninguna de ellas por si misma es capaz de explicar la complejidad humana. Esto implica que la generación de ambientes propicios y el empoderamiento individual son esenciales para crear conductas saludables. Las leyes y regulaciones son un instrumento poderoso, pero no garantizan el cambio individual de hábitos y conductas (61).

Esto es similar a lo que sucedió en las décadas del 60 y 70 en el tema de la desnutrición, donde se planteaba que la única forma de enfrentar y resolver este problema era a través de cambios políticos, socio-económicos y estructurales de los países. Sin embargo, en Chile se demostró que sin una mejoría económica evidente y a través de muy distintos tipos de gobierno que se sucedieron entre las década del 60 y el 80 , políticas muy bien dirigidas desde el sector salud con acciones de prevención primaria (entrega de alimentos a todas las madres y niños que asistían a controles de salud); prevención secundaria( entrega de alimentos focalizados en niños en riesgo de desnutrir o desnutridos leves) y prevención terciaria (rehabilitación en centros especializados) se logró erradicar la desnutrición a fines de la década del 80 (62).

En Chile, el tema de la incorporación de los determinantes, por una parte, desperfiló el trabajo que se venía realizando en cambiar los hábitos y conductas de la población en alimentación, actividad física, tabaco, factores psicosociales y medio ambientales, lo cual ha significado confusión en los grupos técnico-profesionales y en la po-

\section{CUADRO 6}

\section{Documentos relacionados con Promoción de Salud en Chile. 1997- 2006}

1997

1999

1999

2000

2000

2001

2002

2003

2003

2004

2005

2006

2006
Guías de Alimentación para la Población Chile (48)

Plan Nacional de Promoción de Salud (37)

Una Propuesta Comunal de Formación de Recursos Humanos (39)

Metas Intersectoriales al 2006 - VIDA CHILE

Formación de Líderes Comunitarios (40)

Salud sin Tabaco (41)

Objetivos Sanitarios para la década 2000-2010 MINSAL (36)

Guías para una Vida Activa (49)

Consejería de Vida Sana (50)

Guía para una Vida Sana en la Empresa (53)

Guía para una Vida Saludable, Guías Alimentarias, Actividad Física y Tabaco. (51)

EGO ( 52)

Guías para Universidades Saludables (54) 
blación en general. Actualmente, en la política nacional se habla de los determinantes de salud y en los niveles regionales y comunales se continúa trabajando con los condicionantes. Asimismo, al no haber una política coordinada a nivel nacional que dirija en forma consensuada las políticas de Promoción de Salud, han surgido diversas iniciativas separadas unas de otras, como por ejemplo, en alimentación, la estrategia global contra la obesidad (EGO) y la promoción del consumo de frutas y verduras a través de la corporación 5 al día Chile.

Existe además un proyecto de ley en el Congreso para regular el consumo de grasas, azúcares y sal y los alimentos saludables en las escuelas, que se gestó en forma separada de las otras dos iniciativas (63). En actividad física, se creó una red nacional de actividad física, que es parte de la Asociación Latinoamericana de Salud y Actividad Física (64). En tabaco, se aprobó la ley antitabaco en agosto del 2006 y su implementación ha significado una importante campaña a nivel nacional (65). Uno de los grandes logros del funcionamiento de VIDA CHILE Nacional fue que estos temas (alimentación, actividad física; tabaco) eran abordados en forma conjunta y no aislada, como sucede en la actualidad, con lo que lograba un mayor impacto al trabajar en forma integrada.

En consecuencia, después de un avance muy importante en Promoción en Salud en Chile desde 1998 al 2003, la política nacional en esta área se ha ido diluyendo, desarrollándose políticas específicas que hasta el momento no han tenido el impacto que se esperaba, lo que se expresa en la falta de cumplimiento de las metas sanitarias. El desafío es como integrar estas acciones en una política centralizada y coordinada con participación de los sectores gubernamentales, la academia, las estructuras regionales y locales que están trabajando en Promoción de Salud, en conjunto con el ámbito privado. Al igual que lo que sucedió para la erradicación de la desnutrición (58) se requiere de una política de Estado consensuada con financiamiento adecuado, focalizada en los grupos más vulnerables (niños menores de 6 años y escolares hasta la adolescencia) y que sea continua en el tiempo, trascendiendo los gobiernos.

La experiencia en el caso de la desnutrición es que la continuidad de las políticas desarrolladas desde la década del 60 al 80 a través de los más diversos gobiernos, permitió que estas políticas tuviesen éxito. Eso se logró gracias al trabajo conjunto de los grupos de profesionales y técnicos de los diferentes gobiernos con los grupos académicos y profesionales interesados en el tema, que trabajaban apoyando los programas de prevención primaria, secundaria y terciaria. Esta experiencia perfectamente puede ser aplicada en la obesidad, ya que actualmente se cuenta con los recursos humanos capacitados en todos los niveles, con los recursos financieros y con el conocimiento técnico científico necesario para enfrentar los principales factores condicionantes de la obesidad y ECNT que nos afectan.

Para ello disponemos además de una gran cantidad de información recopilada en los últimos años en encuestas sobre calidad de vida y salud que nos permiten tener líneas de base e ir evaluando en forma sistemática los avances en cada uno de los temas, configurando un sistema de vigilancia epidemiológica que permita evaluar y proponer acciones de acuerdo a los resultados que se vayan presentando. Estos insumos para las políticas deberían permitir que una política de Estado consensuada vaya ajustándose a las realidades emergentes y vaya teniendo el impacto requerido para ir acercándonos al cumplimiento de las metas sanitarias al año 2010.

Además, existen experiencias internacionales ampliamente conocidas que podrían replicarse en Chile, como fue la de North Karelia en Finlandia, iniciada en la década del 70. En esta experiencia, en una acción coordinada desde el Estado, se lograron cambiar los hábitos alimentarios de la población desde una alimentación con alto contenido de grasas, azúcar y sal, a uno más saludable de verduras, frutas, pescados, junto con una fuerte regulación de la venta de alimentos procesados, de tabaco y alcohol, mas una política de aumento de las horas de actividad física en las escuelas y facilidades en las empresas para realizarlas. Estas acciones disminuyeron los factores de riesgo de las enfermedades cardiovasculares y cánceres, lo que significó una significativa reducción de la mortalidad en la población finlandesa. Sin embargo, para poder aplicar este tipo de modelos en Chile, hay que considerar el contexto en que se aplicó el modelo finlandés: Finlandia en los años 70 tenía una economía centralmente planificada, con una fuerte injerencia del Estado en la vida de los ciudadanos, lo que hacía que las políticas verticales aplicadas desde el nivel central surtieran efecto en los cambios de hábito de las personas. Chile, desde la década del 80 en adelante, tiene una economía de libre mercado, con una baja ingerencia del Estado en las políticas económicas, con un sistema descentralizado, en que los elementos propios del mercado tienen más influencia en la vida de las personas que lo que pueda hacer el Estado desde sus políticas centralizadas (66).

\section{RESUMEN}

El objetivo de este artículo es analizar las orientaciones técnicas y directrices que han entregado los organismos rectores sobre los factores de riesgo en salud, los cambios en los estilos de vida y su impacto en las 
Enfermedades Crónicas No Trasmisibles (ECNT) en las últimas tres décadas. Se revisan documentos técnicos y directrices de política desde 1978 a la fecha e informes anuales de la Organización Mundial de la Salud en promoción de la salud y de prevención de ECNT. A nivel nacional, se revisan documentos del Ministerio de Salud, (MINSAL), Universidades y entidades relacionadas; se analizan las políticas de promoción de salud y se hacen propuestas nacionales para enfrentar el tema. Se concluye que la estrategia de Promoción de Salud fue incorporada en las políticas nacionales desde 1997, creándose el Consejo Nacional de Promoción de Salud VIDA CHILE en 1998, con avances muy importantes hasta el año 2003, logrando consolidar las acciones de promoción de la salud con VIDA CHILE nacional, VIDA CHILE regionales y VIDACHILE locales en 341 comunas del país. En cada comuna se desarrollaron planes comunales de Promoción de la Salud, con proyectos en alimentación, actividad física, tabaco, temas psicosociales y ambientales. Esto se logró por una alianza estratégica entre el gobierno, principalmente MINSAL, Chiledeportes, Educación, y el sector académico (intersectorialidad). Desde el año 2003, la Promoción de Salud continuó funcionando en el nivel local y regional, en gran medida, por los recursos humanos capacitados en el área, al apoyo del MINSAL y los gobiernos locales. A nivel mundial, en el 2005, en la reunión de Bangkok, la OMS incorpora los determinantes de la salud con el objeto de disminuir las desigualdades en el mundo globalizado, lo que modifica en parte el concepto de promoción de salud, desde un énfasis en cambios de conducta en la población hacia una visión más estructural de los determinantes de salud. Se propone integrar ambos conceptos y desarrollar una Política de Estado semejante a la que se usó para combatir la desnutrición, que sea consensuada y continua en el tiempo, con una alianza estratégica del gobierno, academia y sector privado.

Palabras clave: Promoción de la Salud, factores de riesgo, condicionantes de la salud, enfermedades crónicas no trasmisibles (ECNT), VIDA CHILE.

Dirigir la correspondencia a:

Profesora

Mirta Crovetto M

Facultad de Ciencias Naturales y Exactas

Universidad de Playa Ancha

Valparaíso, Chile

Teléfono: 32-2286713

E-mail:mirtacrovetto@gmail.com

\section{BIBLIOGRAFÍA}

1. ORGANIZACIÓN MUNDIAL DE LA SALUD. $61^{\mathrm{a}}$
Asamblea Mundial de la Salud. En Centro de Prensa 19-24 mayo de 2008. [Online]. Disponible en: http:// www.who.int/mediacentre/events/2008/wha61/es/ index.html [consultado el 09 de diciembre 2008]

2. Organización Mundial de la Salud. Dieta, nutrición y prevención de enfermedades crónicas. Informe de un Grupo de Estudio de la OMS. Ginebra: OMS, 1990.

3. Declaración de Alma Ata. Primera Conferencia Internacional sobre Atención Primaria de Salud en Alma-Ata, Kazajistán, URSS, 6-12 de septiembre de 1978.

4. Carta de Otawa para la Promoción de la Salud. Primera Conferencia Internacional sobre la Promoción de la Salud: Hacia un nuevo concepto de la Salud Pública. Organización Mundial de la Salud. Salud y Bienestar Social de Canadá, Asociación Canadiense de Salud Pública. Notario, Canadá, Noviembre de 1986.

5. Recomendaciones de Adelaida sobre las políticas públicas favorables a la salud. Segunda Conferencia Internacional de Promoción de la Salud: Políticas públicas favorables a la salud. Adelaida, Australia, 5-9 de abril de 1988.

6. Declaración de Sundsvall. Entornos propicios para la salud. Tercera Conferencia Internacional sobre Promoción de la Salud. Organización Mundial de la Salud. Sundsvall (Suecia), 9-15 de junio de 1991

7. Declaración de Bogotá. Promoción de la Salud en América Latina. Conferencia Internacional de Promoción de la Salud. Santa Fe. Bogota, Noviembre 1992.

8. Actas del Primer Seminario Internacional sobre políticas, estrategias, estructuras en Promoción de la Salud Instituto Nacional de Salud. París, Francia, del 21 al 23 Noviembre, 1994.

9. Declaración de Yakarta sobre Promoción de la Salud en el siglo XXI. Cuarta Conferencia Internacional sobre Promoción de la Salud: Nueva era, nuevos actores: Adaptar la Promoción de la Salud al siglo XXI. Organización Mundial de la Salud, Yakarta República de Indonesia, 21-25 de julio de 1997.

10. 51 ava Asamblea Mundial de la Salud. Organización Mundial de la Salud. WHA51.12 Punto 20 del orden del día 16 de mayo de 1998 Promoción de la Salud. Washington, 1998.

11. Declaración de México hacia una mayor equidad. Quinta Conferencia Internacional sobre Promoción de la Salud hacia una mayor equidad. Organización Mundial de la Salud. Organización Panamericana de la Salud. Santa Fe, México, 5-9 Junio 2000.

12. Carta de Bangkok para el Fomento de la salud en 
un mundo globalizado. VI Conferencia Mundial de Promoción de la Salud. Tailandia, Bangkok, 7-11 Agosto 2005.

13. Informe sobre la salud en el mundo 1995 - Reducir las desigualdades. Organización Mundial de la Salud. Ginebra, 1995.

14. Informe sobre la salud en el mundo 1996-Combatir las enfermedades, promover el desarrollo. Organización Mundial de la salud. Ginebra, 1996.

15. Informe sobre la salud en el mundo 1997 - Vencer el sufrimiento, enriquecer a la humanidad. Organización Mundial de la Salud. Ginebra, 1997.

16. Informe sobre la salud en el mundo 1998 - La vida en el siglo XXI. Organización Mundial de la Salud. Ginebra, 1998.

17. Informe sobre la salud en el mundo 1999 - Cambiar la situación. Organización Mundial de la Salud. Ginebra 1999.

18. Informe sobre la salud en el mundo 2000 - Mejorar el desempeño de los sistemas de salud. Organización Mundial de la Salud. Ginebra 2000.

19. Informe sobre la salud en el mundo 2002 - Reducir los riesgos y promover una vida sana. Organización Mundial de la Salud. Ginebra, 2002.

20. Informe sobre la salud en el mundo 2003 - Forjemos el futuro. Organización Mundial de la Salud. Ginebra, 2003.

21. El Informe sobre la salud en el mundo 2006 - Colaboremos por la salud. Organización Mundial de la Salud. Ginebra, 2006

22. Informe sobre la salud en el mundo 2007 - Un porvenir más seguro Protección de la salud pública mundial en el siglo XXI. Organización Mundial de la Salud. Ginebra, 2007

23. Informe sobre la salud en el mundo 2008. Revigorización de la atención primaria de salud. En prensa. Organización Mundial de la Salud. Ginebra, 2008

24. Organización Mundial de la Salud. Dieta, nutrición y prevención de enfermedades crónicas. Informe Técnico No 797. Ginebra: OMS; 1990.

25. FAO/OMS. Conferencia Internacional sobre Nutrición. Informe final de la Conferencia. Roma 1992.

26. Organización Mundial de la Salud. Serie de Informes Técnicos, TRS, 916, 2002

27. Organización Mundial de la Salud. Estrategia Mundial sobre Régimen Alimentario, Actividad Física y Salud. Ginebra, 2005.

28. Las Américas libres de grasas trans, conclusiones y recomendaciones para la OPS/OMS 28 y $27 \mathrm{de}$ abril de 2007, Washington, D.C

29. $4^{\circ}$ Simposio Internacional del Programa Promoción del Consumo de Frutas y Verduras, realizado en Christchurch, Nueva Zelanda 2004.

30. World Cancer Research Fund/American Institute for Cancer Research. Food, Nutrition, Physical Activity, and the Prevention of Cancer: a Global Prespective. Washington, DC: AICR, 1997.

31. World Cancer Reseach Fund/American Institute for Cancer Research. Food, Nutrition, Physical Activity, and the Prevention of Cancer: a Global Perspective. Washington, DC: AICR, 2007.

32. Albala C, Vio F, Robledo A, Icaza G. La transición epidemiológica en Chile. Rev Med Chil 1993; 121: 1446-1455

33. Albala C, Vio F. Epidemiologic transition in Latin America. The case of Chile. Public Health 1995; 109: 431-442

34. Vio F, Albala C. La transición nutricional en Chile. Rev Chil Nutr 1998; 25(3): 11-20

35. Ministerio de Salud. Estudio de Prioridades de Inversión en Salud. Estudio Carga de Enfermedad. Informe Final, Marzo 1996.Estudio de Carga de Enfermedad 1993. Pérdida de Años de Vida, ajustados por discapacidad (AVISA) Chile, 1993.

36. Ministerio de Salud. División de salud de las personas. Diseño e implementación de las prioridades de salud: la reforma programática chilena: 1997. [Santiago de Chile]:

37. Ministerio de Salud. División de Atención Primaria.. Unidad de Promoción de la Salud. Plan nacional de Promoción de la Salud. 1999.

38. Ministerio de Salud. Boletín de Vigilancia en Salud Pública de Chile El Vigía, Resumen Ejecutivo Objetivos Sanitarios para la década 2000-2010 Vol 5, $\mathrm{N}^{\mathrm{o}} 15$, abril 2002

39. F. Vio, M C, G Yury, M Castillo. Programa de Formación de Recursos Humanos en Promoción de la Salud. Una Propuesta Comunal. Universidad de Chile, INTA: Ministerio de Salud; Santiago, Chile, 1999.

40. M Crovetto, F Vio, M Castillo, G Yury. Programa de Formación de Recursos Humanos en Promoción de la Salud. Formación de Líderes Comunitarios. Universidad de Chile, INTA: Ministerio de Salud: Santiago, Chile, 2000.

41. Gobierno de Chile. Ministerio de Salud. Salud sin tabaco. División de Atención Primaria. Departamento Promoción de la Salud, Programa Ambientes Libres del Humo de Tabaco.Guía Técnica- Metodológica. Programa Ambientes Libres del Humo de Tabaco. Noviembre 2001.

42. Acta de Huechuraba: Por un país más saludable, Santiago, agosto 1999. 
43. Libro de Resúmenes I Congreso Chileno de Promoción de la Salud 24 al 27 agosto 1999.

44. Libro de Resúmenes de Trabajos. II Congreso Chileno de Promoción de la Salud, 24- 26 de octubre 2002.

45. Ministerio de Salud. Instituto Nacional de Estadística. I Encuesta Nacional de Calidad de Vida. Santiago, Chile, 2000.

46. Ministerio de Salud. Instituto Nacional de Estadísticas. Encuesta de Calidad de Vida y Salud 2006. Departamento de Epidemiología y Departamento de Promoción de Salud. Santiago, 2007.

47. Ministerio de Salud. Encuesta Nacional de Salud 2003. (ENS). Informe final. Santiago, Chile: MINSAL, 2004 ENS.

48. Ministerio de Salud. Guías de Alimentación Para la Población Chilena. C. Castillo, R. Uauy, E. Atalah eds. Ed. Diario La Nación, Santiago de Chile, 1997

49. Ministerio de Educación, Ministerio de Salud, Chile Deportes, INTA. Guía para una Vida Activa. Vio F, Salinas J, Eds. Andros Impresores. Santiago, Chile 2003.

50. INTA, MINSAL. Modelos de intervención promocional en centros de atención primaria: Consejerías de Vida Sana, Santiago, Chile 2004.

51. INTA, Ministerio de Salud. Guía para una Vida Saludable, Guías Alimentarias, Actividad Física y Tabaco. Andros Impresores, Santiago, Chile 2005.

52. Gobierno de Chile. Ministerio de Salud. Estrategia Global contra la Obesidad (EGO-Chile). Documento de Trabajo, diciembre 2005.

53. Calderón B, Salinas J, Vio F. Eds. Consultoras: H Grumpeter, M Parra. Vida sana en la empresa. Guía práctica para empresas. Santiago: Ministerio de Salud, MINSAL / INTA Universidad de Chile / Consejo Nacional VIDA CHILE / Acción RSE. 2004.

54. Guía para Universidades Saludables y otras instituciones de Educación Superior. I. Lange, Vio F, Eds. Andros Impresores. Santiago, Chile 2006.

55. Atalah E, Castillo C, Castro R, Aldea A. Propuesta de un nuevo estándar de evaluación nutricional en embarazadas. Rev Med Chil. 1997; 125: 14291436.

56. Encuesta Nacional de Hábitos de Actividad Física y Deportes. CHILE DEPORTES,2006
57. Séptimo Estudio Nacional de Drogas en población escolar de Chile, 2007, de 8avo básico a cuarto medio. Principales resultados, Junio 2008.

58. Kain J, Uauy R, Leyton B, Cerda R, Olivares S, Vio F. Efectividad de una intervención en educación alimentaria y actividad física para prevenir obesidad en escolares de la ciudad de Casablanca, Chile (20032004). Rev Med Chil 2008; 136:22-30.

59. CJMurray,ADLopez-Lancet, 1997-ncbi.nlm.nih.gov Lancet. 1997 May 3;349(9061):1269-76. Mortality by cause for eight regions of the world: Global Burden of Disease Study.

60. Editorial. From what we will die in 2020. The Lancet 1997; 349:1263

61. 61 Fuentes A Aporte de la psicología y sociología a la Promoción de la Salud en Chile. Cuad Med Soc 2008; 48:118-124

62. 62. Vio F, Weisstaub G, Atalah E, Boj T, Jiménez M, Fernández P, Puentes G, Fuccaro D, Rojas J, Vásquez M, Folch J, García G, Méndez G. Desnutrición infantil en Chile: Políticas y Programas que explican su erradicación. Conferencia Ministerial Regional "Hacia la erradicación de la desnutrición Infantil en América Latina y el Caribe”. Santiago, abril 2008.

63. 63.GIRARDI, GUIDO. Proyecto de Ley sobre regulación de alimentos poco saludables. En: Actualidad legislativa, 18 de marzo 2008 [Online]. Disponible en:http://www.bcn.cl/actualidad_legislativa/temas_portada.2008-03-17.9309833535/4921_11.pdf [Consultado el 09 de diciembre 2008]

64. Matsudo SM, Matsudo VR, Andrade DR et al. Physical activity promotion: experiences and evaluation of the Agita Sao Paulo program using the ecological mobile model. J Phys Act Health 2004; 1: 81-97.

65. CHILE. MINISTERIO DE SALUD PUBLICA. LEY-19419 de 1995. Regula actividades que indica relacionadas con el tabaco. Disponible en: http:// www.minsal.cl/ici/tabaco/LEY_19419.doc [Consultado el 09 de diciembre 2008]

66. Puska P Tuomilehto J, Nissinen A, Vartiainen E. eds. The North Karelia Project.

67. 20 years results and experiences. Printed by: Helsinski University Printing

68. House. National Public Health Institute, KTL, Helsinki 1995. 